\title{
Data Flow in Wireless Sensor Network Protocol Stack by using Bellman-Ford Routing Algorithm
}

\author{
Rakesh Kumar Saini ${ }^{\star 1}$, Ritika $^{2}$, Sandip Vijay ${ }^{3}$ \\ ${ }^{1,2}$ Department of Computer Science and Application, DIT University, Dehradun, Uttrakhand, India \\ ${ }^{3}$ Department of Electronics and Communication, ICFAI University, Dehradun, Uttrakhand, India \\ ${ }^{*}$ Corresponding author, e-mail: rk.saini@dituniversity.edu.in ${ }^{1}$, hod.mca@dituniversity.edu.in ${ }^{2}$, \\ vijaysandip@gmail.com ${ }^{3}$
}

\begin{abstract}
Wireless sensor network consists various sensor nodes that are used to monitor any target area like forest fire detection by our army person and monitoring any industrial activity by industry manager. Wireless sensor networks have been deployed in several cities to monitor the concentration of dangerous gases for citizens. In wireless sensor network when sensor nodes communicate from each other then routing protocol are used for communication between protocol layers. Wireless sensor network protocol stack consist five layers such as Application layer, Transport layer, Network layer, MAC Layer, Physical layer. In this paper we study and analysis Bellman-Ford routing algorithm and check the flow of data between these protocol layers. For simulation purpose we are using Qualnet 5.0.2 simulator tool.
\end{abstract}

Keywords: sensor nodes, wireless sensor network, Bellman-Ford routing algorithm, Qualnet 5.0.2

\section{Introduction}

A Wireless sensor network has many sensor nodes that are interconnected by wireless communication channels. Sensor node is a small device that is used to monitor the physical and environmental condition such as temperature, sound and any movement in the sensing field. Sensor node senses the data and sends to nearest sensor node and all sensor nodes cooperately pass their data to the base station via internet or satellite. Nowadays wireless sensor networks are popular and their applications are used by our military for environment monitoring. Wireless sensor networks are used for other purpose like home security, earthquake warning. Despite the infinite scopes of wireless sensor networks, they are limited by the node battery lifetime. Once they are deployed, the network can keep operating while the battery power is adequate. This is critical point to be considered as it is almost impossible to replace the node battery once deployed over an inaccessible area. Such constraints combined with a typical deployment of large number of sensor nodes, have posed many challenges to the design and management of sensor networks and necessitate energy-awareness at all layers of networking protocol stack [1-2]. The main task of a sensor node in a sensor field is to detect events, perform quick local data processing, and then transmit the data. Power consumption can hence be divided into three domains: sensing, communication, and data processing. In this paper we study and analysis Bellman-Ford routing algorithm and check the flow of data between Wireless sensor network protocols stack layers.

\section{Wireless Sensor Network}

A wireless sensor network consists distributed autonomous sensors nodes that are used to monitor physical or environmental conditions, such as temperature, sound, vibration, pressure and to cooperatively pass their data to the base station [2-3]. The more modern networks are bi-directional, also enabling control of sensor activity. The development of wireless sensor networks was motivated by military applications such as battlefield surveillance; today such networks are used in many industrial and consumer applications, such as industrial process monitoring and control machine health monitoring, and so on. The WSN is built of "nodes" - from a few to several hundreds or even thousands, where each node is connected to one (or sometimes several) sensors. Each such sensor network node has typically several 
parts: a radio transceiver with an internal antenna or connection to an external antenna, a microcontroller, an electronic circuit for interfacing with the sensors and an energy source, usually a battery or an embedded form of energy harvesting. A sensor node might vary in size from that of a shoebox down to the size of a grain of dust, although functioning "motes"(demo video) of genuine microscopic dimensions have yet to be created. The cost of sensor nodes is similarly variable, ranging from hundreds of dollars to a few pennies, depending on the complexity of the individual sensor nodes. Size and cost constraints on sensor nodes result in corresponding constraints on resources such as energy, memory, computational speed and communications bandwidth. The topology of the WSNs can vary from a simple star network to an advanced multi-hop network. The wireless sensor node, being a microelectronic device, can only be equipped with a limited power source ( $<0.5 \mathrm{Ah}, 1.2 \mathrm{~V})$. In some application scenarios, replenishment of power resources might be impossible [5]. Sensor node lifetime, therefore, shows a strong dependence on battery lifetime. In a multihop ad hoc sensor network, each node plays the dual role of data originator and data router. The malfunctioning of a few nodes can cause significant topological changes and might require rerouting of packets and reorganization of the network. Hence, power conservation and power management take on additional importance [4-5]. It is for these reasons that researchers are currently focusing on the design of power-aware protocols and algorithms for sensor networks. In Wireless Sensor network, all sensor nodes move from one location to another location [7]. In this paper we analyze Bellman-Ford routing algorithm and check the data flow between protocol layers [9].

\section{Protocol Stack of WSN}

Wireless sensor network protocol stack has five layers such as Application layer, Transport layer, Network layer, MAC Layer, Physical layer [10-11].The Wireless sensor network protocol stack used by base station and sensor nodes as mentioned in Figure 1 (Wireless Sensor Network Protocol Stack). WSNs Protocol Stack combines energy of sensor nodes and routing awareness, integrates packets with networking protocols, communicates energy efficiently through the wireless medium, and promotes cooperative efforts of sensor nodes. Wireless sensor network protocol stack is modification of OSI (Open system interconnection). It is used for wireless network. In wireless sensor network when sensor nodes cooperately pass their data to the base station then this protocol stack works for exchanging or passing information from one layer to another layer adjacently [12]. It does not provide communication non-adjacently between Protocol layers.

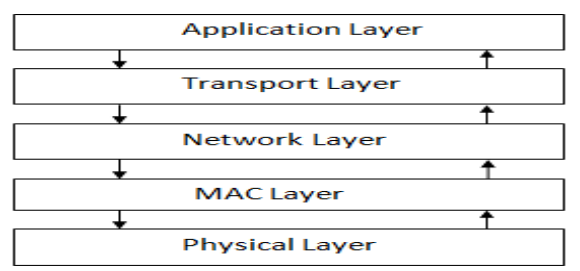

Figure 1. Wireless Sensor Networks protocol stack

In WSNs, The physical layer is responsible for frequency selection, carrier frequency generation, signal detection, modulation, and data encryption. The MAC layer is responsible for the multiplexing of data streams, data frame detection, medium access and error control. It ensures reliable point-to-point and point-to-multipoint connections in a communication network. The network layer takes care of routing the data supplied by the transport layer. The transport layer helps to maintain the flow of data if the sensor networks application requires it. Depending on the sensing tasks, different types of application software can be built and used on the application layer. In addition, the power, mobility, and task management planes monitor the power, movement, and task distribution among the sensor nodes. These planes help the sensor nodes coordinate the sensing task and lower overall power consumption. The power management plane manages how a sensor node uses its power. 


\section{Simulation Setup}

For analysis of data flow between WSNs protocol stack layers we create a simulation setup as shown in Figure 2. In this simulation setup we consider 17 sensor nodes that are communicate between each other and finaly send data to destination node. In this simulation we use Bellman-Ford routing algorithm for routing data packets between sensor nodes. Bellman-Ford routing algorithm computes shortest paths from sensor node to all other sensor nodes and finally sends data packets by a shortest path to the base station. Bellman-Ford routing algorithm is very usefull and energy-efficient approach in wireless sensor network. Running simulation Figure 3 show the working of all sensor nodes [13-14]. There are many sensor nodes communicate from each other and finally send cooperately sense data to destination nodes. In this simulation we are using Qualnet 5.0.2 simulator tool. Qualnet 5.0.2 is created for Wireless sensor network and Adhoc network.

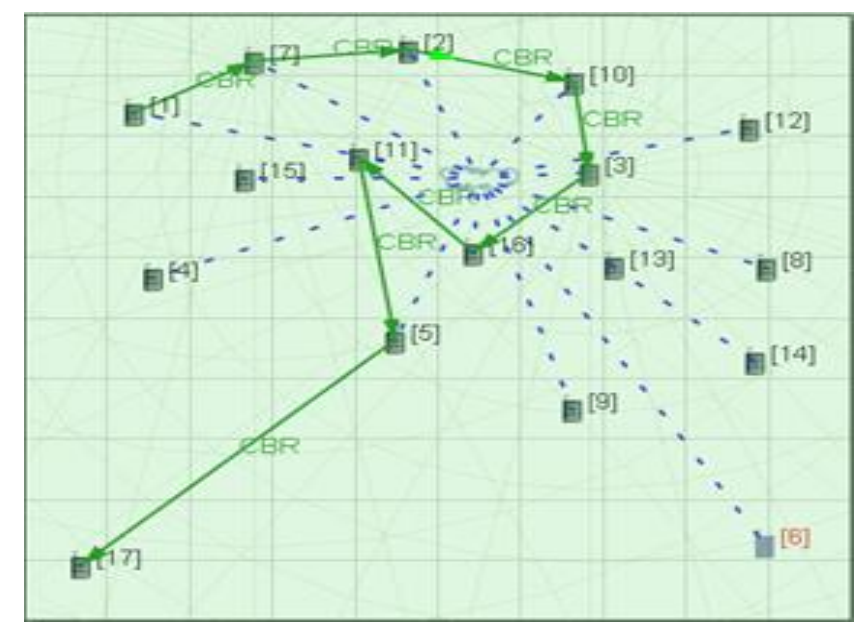

Figure 2. Simulation setup

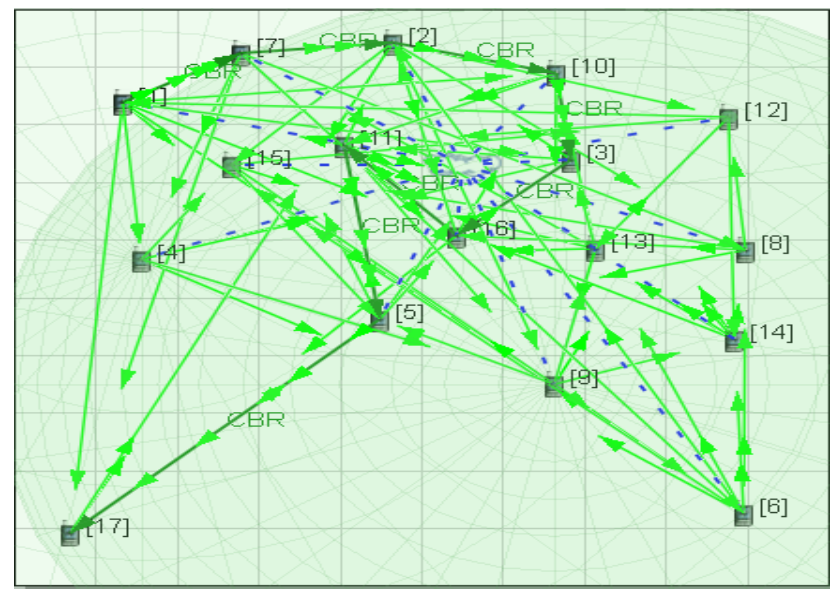

Figure 3. Running simulation

\section{Simulation Results and Analysis}

For Study and analysis of data packets from top layer (Application layer) to bottom layer (Physical layer) we are consider Qualnet 5.0.2 simulator tool. Qualnet 5.0.2 is a very popular simulator tool in wireless sensor network. Parameters that are used in this simulation are shown in Table 1. In this simulation the performance of Bellman-Ford is verified. In this simulation we consider sensor nodes $1,2,3,5,7,10,11,16$ cooperately pass their data to the destination node 17. 
Table 1. Simulation Parameters

\begin{tabular}{ll}
\hline \multicolumn{1}{c}{ Parameter } & \multicolumn{1}{c}{ Value } \\
\hline Simulator & Qualnet 5.0 .2 \\
Source Sensor nodes & $1,2,3,5,7,10,11,16$ \\
Destination node & 17 \\
Buffer Size & 1024 \\
Terrain Range & $100 \mathrm{~m} \times 100 \mathrm{~m}$ \\
No. of sensor nodes & 17 \\
Frequencies & $2.4 \mathrm{GHz}$ \\
Traffic Type & CBR \\
Channel Type & Wireless channel \\
Protocols & BELLMAN-FORD \\
\hline
\end{tabular}

In this simulation for study and analysis of Bellman-Ford routing algorithm we check the values on wireless sensor network protocol stack (Application layer, Transport layer, Network layer, MAC layer, Physical layer). First we check data packets that are coming from Application layer to Transport layer similarly flow of data packets from Transport layer to Network layer, Network layer to MAC layer and from MAC layer to Physical layer [17-18].

In Figure 4, Data packets send from Application layer to Transport layer In Figure 5, Data packets received from Application layer to Transport layer. In Figure 6 Data packets received from Transport layer to Network layer. In Figure 7. Data packets received from Network layer to MAC layer. In Figure 8 Data packets received from MAC layer to Physical layer.

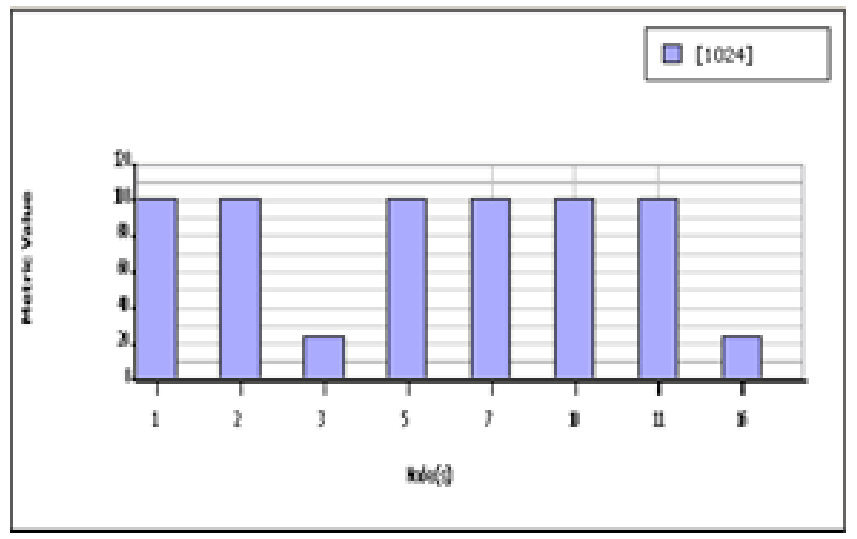

Figure 4. Data packets send from application layer to transport layer

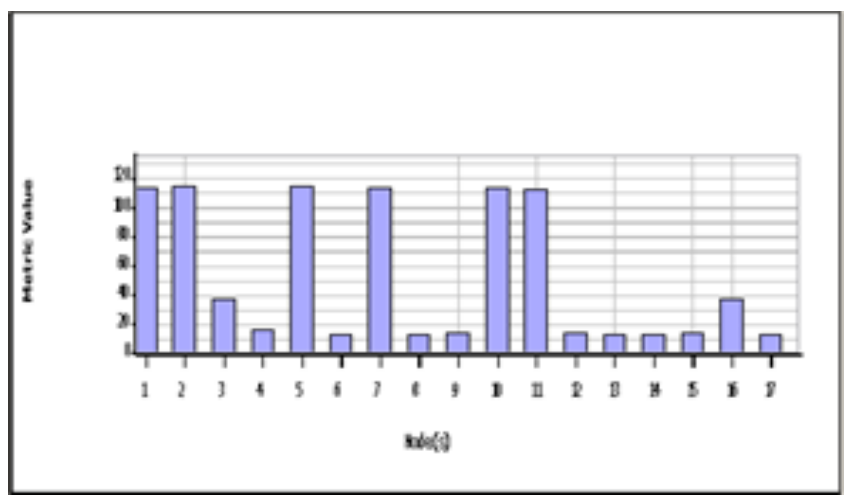

Figure 5. Data packets received from application layer at transport 


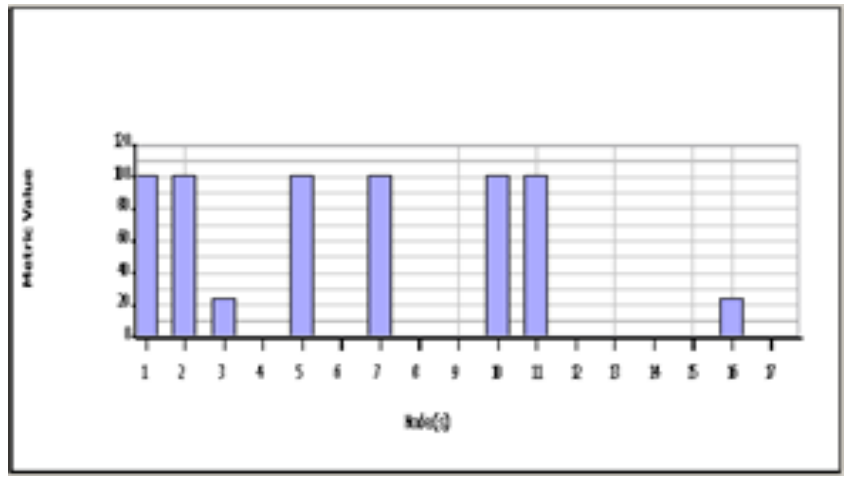

Figure 6. Data packets received from transport layer at network

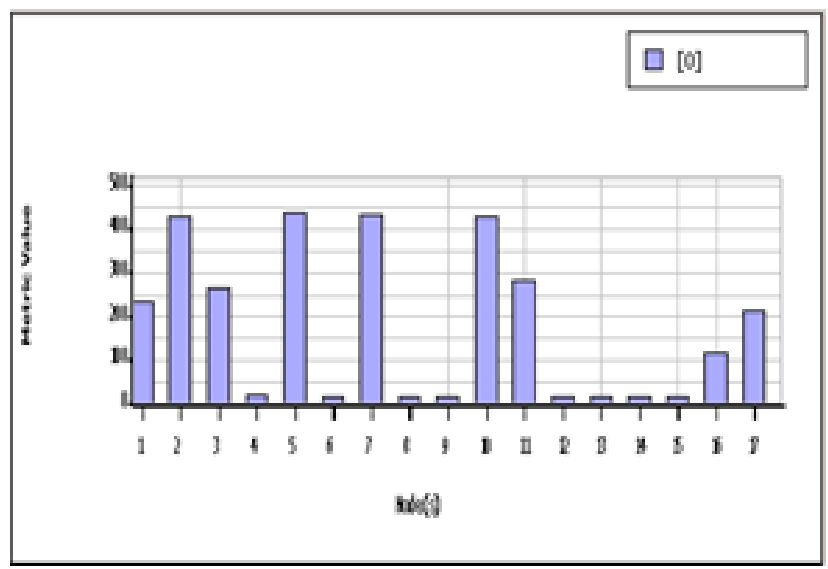

Figure 7. Data packets received from network layer at MAC layer

In this simulation result, data packets that are coming from Transport layer to Network layer show frequency of sensor nodes adjacently. Similarly data packets coming from Network layer to MAC layer and then data packets are coming from MCA layer to Physical layer. By study and analysis of data packets flow from top layer(Application layer) to Bottom layer(Physical layer) we find that all sensor nodes forwarding data to the base station adjacently means one layer to next layer and so on. By using Bellman-Ford routing algorithm all sensor nodes forward data to base station but Bellman-Ford routing algorithm is not energy-efficient [15]. For improve energy-efficiency in wireless sensor network we can use Cross-layer design approach in wireless sensor network. Cross-layer design approach is more energy-efficient, scalable and useful approach in wireless sensor network [16].

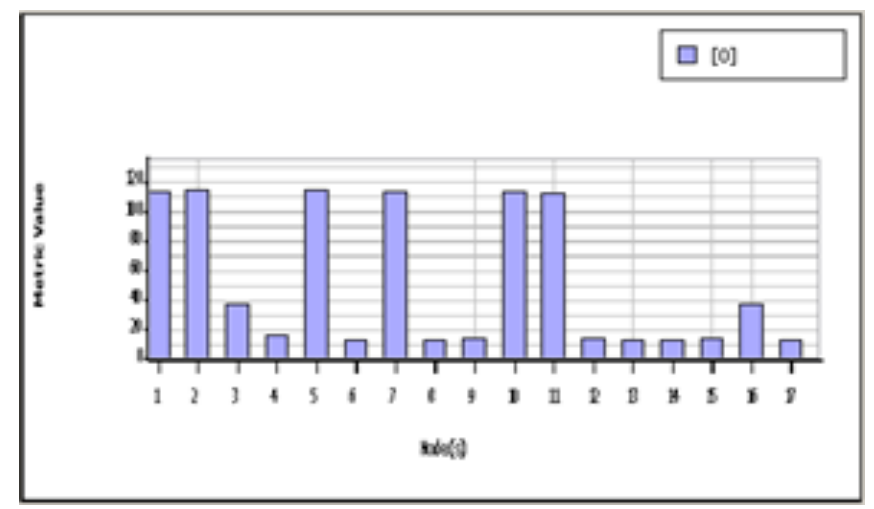

Figure 8. Data Packets Received from MCA Layer at Physical Layer 


\section{Conclusion}

In this simulation we study and analysis of Bellman-Ford routing algorithm at different wireless sensor network protocol stack layer (Application layer, Transport layer, Network layer, MAC layer, Physical layer). These layers exchange data adjacently between each other. In this simulation we analysis data flow at different-different layer. In future work we will develop a cross-layer design approach that will be more energy-efficient as compare to traditional layered approach.

\section{References}

[1] IF Akyildiz et al., Wireless sensor networks: a survey, Computer Networks 38. 2002: 393-422.

[2] JN Al-Karaki, AE Kamal. Routing Techniques in Wireless Sensor Networks: A Survey. IEEE Wireless Communication. Dec. 2004; 11(6): 6-28.

[3] WB Heinemann et al., An Application-Specific Protocol Architecture for Wireless Micro sensor Networks. IEEE Transactions on Wireless Communications. Oct 2002; 1(4): 660 - 670.

[4] WR Heinemann, A Chandrakasan, H Balkrishnan. Energy-Efficient Communication Protocol for Wireless Micro sensor Networks. In Proceedings of 33rd Hawaii International Conference on System Science. Jan. 2000; 2: 1-10.

[5] Mao Ye, Chengfa Li, Guihai Chen, Jie Wu. An Energy efficient clustering scheme in Wireless sensor network. Ad Hoc and Sensor wireless Networks. April 2006; 3: 99-119.

[6] Amir Sepasi Zahmati, Bahman Abolhassani, Ali Asghar Behesti Shirazi, Ali Shojaee Bakhtiari. An Energy-Efficient protocol with Static clustering for Wireless Sensor Network. Proceedings of world academy of science, Engineering and Technology. July 2007; 22.

[7] C-Y Chong, SP Kumar. Sensor networks: evolution, opportunities, and challenges. Proceedings of the IEEE. 2003; 91(8): 1247-1256.

[8] Soheil Ghiasi, Ankur Srivastava, Xiaojian Yang, Majid Sarrafzadeh. Optimal Energy Aware Clustering in Sensor Networks. SENSORS Journal. 2002; 2(7): 258-269.

[9] Al-Karaki, Jamal N, Ahmed E Kamal. Routing techniques in wireless sensor networks:a survey. Wireless communications, IEEE. 2004; 11(6): 6-28.

[10] Mardani, Morteza, Seung-Jun Kim, Georgios B Giannakis. Cross-layer design of wireless multihop random access networks. Signal Processing, IEEE Transactions. 60.5 (2012): 2562-2574.

[11] Heinemann, Wendi Rabiner, Anantha Chandrakasan, Hari Balakrishnan. Energy-efficient communication protocol for wireless micro sensor networks. System sciences, 2000. Proceedings of the 33rd annual Hawaii international conference on. IEEE. 2000.

[12] [Herman Sahota, Ratnesh Kumar, Ahmed Kamal, Jing Huang. An Energy-Efficient Wireless Sensor Network for Precision Agriculture. In Proc. IEEE. 2010.

[13] Yong Ding, Zhou Xu, Lingyun Tao. An Cross Layer Algorithm Based on Power Control for Wireless Sensor Networks. Springer-Verlag Berlin Heidelberg. 2010: 209-216.

[14] Qingxu Xiong, Xiang. Cross-layer design of MAC and application semantics in Wireless Sensor Netowrks. IEEE 2014 fourth international conference on communication systems and network technologies.

[15] Atif Sharif, Vidyasagar M Potdar, AJD Rathnayaka. LCART: A Cross-layered Transport Protocol for Heterogeneous WSN. IEEE SENSORS 2010 Conference.

[16] lan F.Akyildiz, Mehmet CVuran, Ozgur B Akan. A Cross-Layer Protocol for Wireless Sensor Network. In Proc. IEEE 2006.

[17] Chia-Hung Tsai, Tsu-Wen Hsu, Meng-Shiuan Pan, Yu-Chee Tseng. Cross-Layer, Energy-Efficient design for supporting continuous queries in Wireless Sensor Network: A Quorum-Based Approach. Springer Science+Business Media, LLC. 2009.

[18] Huaqing Wang, Yue Ouyang, Guixia Kang. An Energy Efficient Cross-Layer Design for Healthcare Monitoring Wireless Sensor Networks. In Proc.IEEE 2008.

[19] Thomas Beluch, Daniela Dragomirescu, Robert Plana. A Sub-nanosecond Synchronized MAC-PHY cross-layer design for Wireless Sensor Networks. ELSEVIER, Ad Hoc Networks. 2013; 11: 823-845. 